\title{
Emerging Issues and Opportunities in Health Information Technology
}

\author{
Elizabeth A. Nardi, MS'; Lisa Korin Lentz, MPHª Katherine Winckworth-Prejsnar, MPHa;
} Amy P. Abernethy, MD, $\mathrm{PhD}^{\mathrm{b}}$; and Robert W. Carlson, $\mathrm{MD}^{\mathrm{a}}$

\begin{abstract}
When used effectively, health information technology (HIT) can transform clinical care and contribute to new research discoveries. Despite advances in HIT and increased electronic health record adoption, many challenges to optimal use, interoperability, and data sharing exist. Data standardization across systems is limited, and scanned medical note documents result in unstructured data that make reporting on quality measures for reimbursement burdensome. Different policies and initiatives, including the Health Information Technology for Economic and Clinical Health Act, the Medicare Access and CHIP Reauthorization Act, and the National Cancer Moonshot initiative, among others, all recognize the impact that HIT can have on cancer care. Given the growing role HIT plays in health care, it is vital to have effective and efficient HIT systems that can exchange information, collect credible data that is analyzable at the point of care, and improves the patient-provider relationship. In June 2016, NCCN hosted the Emerging Issues and Opportunities in Health Information Technology Policy Summit. The summit addressed challenges, issues, and opportunities in HIT as they relate to cancer care. Keynote presentations and panelists discussed moving beyond Meaningful Use, HIT readiness to support and report on quality care, the role of HIT in precision medicine, the role of HIT in the National Cancer Moonshot initiative, and leveraging HIT to improve quality of clinical care.
\end{abstract}

J Natl Compr Canc Netw 2016;14(10):1226-1233

\section{Executive Summary}

Health information technology (HIT) has been directly or indirectly the subject matter of many government initiatives or legislative matters during the past decade. The Health Information Technology for Economic and Clinical Health (HITECH) Act, the Medicare Access and CHIP Reauthorization Act (MACRA), and the National Cancer Moonshot initiative, among others, all recognize the impact HIT can have on cancer care. Given the growing body of data, including genomic data, it is vital to have effective HIT systems that can meaningfully interpret and exchange information in an effort to enhance care quality and clinical outcomes. Despite advances in HIT and increased electronic health record (EHR) adoption, many challenges to optimal use, interoperability, and data sharing exist.

Furthermore, some studies have suggested that there are significant challenges and opportunities for using HIT in cancer care in particular. ${ }^{1}$ Cancer care

From a National Comprehensive Cancer Network, Fort Washington, Pennsylvania, and 'Duke University Medical Center, Cary, North Carolina.

Submitted August 16, 2016; accepted for publication September 22, 2016. is unique in that it often involves patient transitions across multiple providers and is also moving toward markedly personalized medicine based on biomarker data and individual patient preferences. Given the multidisciplinary approach to cancer, coordinating multiple specialties' patient information across systems is essential to ensuring high-quality and efficient care; contributing to a more satisfactory patient experience; and easing administrative burden on data exchange and clinical interpretation of information. Thus, the ability to readily share and reconcile information and make effective, personalized medical decisions relies heavily on the HIT infrastructure storing each patient's unique information at each provider as well as on the availability of clinical decision support tools, which are based on rapidly evolving clinical guidelines.

In June 2016, NCCN hosted a Policy Summit to address the challenges, issues, and opportunities in HIT as they relate to cancer care. Keynote presentations and

Ms. Nardi, Ms. Lentz, Ms. Winckworth-Prejsnar, and Dr. Carlson are employees of NCCN. Dr. Abernethy has disclosed that she is employed by Flatiron Health.

Correspondence: Elizabeth A. Nardi, MS, NCCN, 275 Commerce Drive, Suite 300, Fort Washington, PA 19034. E-mail: nardi@nccn.org 
panelists discussed moving beyond Meaningful Use, HIT readiness to support and report on quality care, the role of HIT in precision medicine, the role of HIT in the National Cancer Moonshot initiative, and leveraging HIT to improve quality of clinical care. The summit sought to address the HIT challenges and opportunities across a variety of stakeholders and included perspectives from clinicians, patients, government, private payers, academia, and HIT organizations.

\section{From Meaningful Use to Advancing Care Information}

In 2009, fewer than 20\% of physicians and hospitals used basic EHRs; that number today has increased to $99 \%$ of hospitals and $74 \%$ of physicians. ${ }^{2}$ The Centers for Medicare \& Medicaid Services' (CMS) EHR Incentive Program accelerated this progress. The program, part of a broader effort under the HITECH Act to increase the adoption of HIT and use of qualified EHRs, established incentive payments to eligible professionals, eligible hospitals, critical access hospitals, and Medicare Advantage organizations to promote the adoption and meaningful use of interoperable HIT and qualified EHRs. ${ }^{3}$ As a result, more than 300,000 professionals received incentive payments. ${ }^{1}$

Although these data demonstrate widespread HIT adoption across clinicians in general, many cancer care providers are not participating in the EHR Incentive Program. According to a CMS report, only $2 \%$ of the Medicare EHR Incentive Program payments made between 2011 and 2013 were made to oncologists and hematologists. ${ }^{4}$ Furthermore, according to the CMS 2014 Physician Quality Reporting System (PQRS) Experience Report, only 8\% of oncologists, $2 \%$ of nuclear medicine physicians and radiation oncologists, and $1 \%$ or less of interventional radiologists, radiologists, and pathologists participated via EHR reporting. ${ }^{5}$ These data show that there is room for increased EHR adoption in oncology care, and call into question why EHR acceptance has not been as widespread as in other clinical specialties.

Despite significant progress since the HITECH Act, the advances and ease of use of technology in the health care sector still lags behind technological advances and effective use seen in other industries.
Many HIT systems lack interoperability, thus impeding digital communication within and across systems; documents regarding care received at one medical institution are faxed over to another medical institution and scanned into a new system in PDF form as unstructured data. ${ }^{6}$ Further, there is a limited range of performance data and significant reporting burden on providers to document the care delivered. Recognizing the need for continued use and improvement of EHR and HIT systems, CMS announced in early 2016 that it will end the EHR Incentive Program and roll it into provisions set forth in MACRA. Under MACRA, eligible clinicians (ECs) will still be held accountable for incorporating information technology into their practices.

In early 2015, the U.S. Department of Health and Human Services (HHS) announced new goals for value-based payments and alternative payment models (APMs) in Medicare, and later that year, Congress passed the MACRA. Under the legislation, ECs receive payment by participating in the Merit-based Incentive Payment System (MIPS) or Advanced Alternative Payment Models. MIPS streamlines 3 quality and value reporting programs for Medicare providers (PQRS, Value-Based Payment Modifier, and EHR Incentive Program) to work as one, with a fourth component that promotes ongoing improvement and innovation. A single MIPS composite performance score, using a 100-point scale, will factor in performance in 4 weighted categories: Quality, Resource Use, Clinical Practice Improvement Activities, and Advancing Care Information. Most ECs in year one, 2017, will be subject to MIPS and scored on these categories. The composite score will determine how providers are reimbursed.

Of these 4 performance categories, the Advancing Care Information (ACI) category emphasizes patient engagement, interoperability, information exchange, and security. CMS believes the ACI performance category is more customizable than the EHR Incentive Program because it allows providers to report the measures that are most meaningful to their practice. CMS proposes 6 objectives and related measures that would require reporting for this category; some objectives include patient electronic access, coordination of care through patient engagement, and health information exchange. MACRA also requires eligible providers to demonstrate that 
Nardi et al

they have not knowingly or willingly limited or restricted interoperability, and to cooperate with HIT surveillance to ensure that technology needs meet federal standards.

Despite great progress in the adoption of EHR use, many issues exist, particularly in cancer care. Interoperability, lack of data standardization, and reporting burdens for providers remain significant challenges to achieving high-quality care. One area in which HIT may lag behind for oncology and cancer care as opposed to other specialties and conditions is in clinical decision support. Many HIT vendors are in the beginning stages of licensing and implementing cancer care guidelines and pathways in their EHR systems, whereas CMS recently proposed retiring the Clinical Decision Support measure from the EHR Incentive Program, stating that performance on this measure has been high and no longer contributes to HIT-related objectives. ${ }^{\text {? }}$

Nevertheless, recent reforms aim to mitigate these challenges while continuing to promote the use of HIT and EHRs. As payment models move from volume to value, the use, and ease of use, of HIT becomes crucial for success.

\section{Interoperability: The Path Forward}

The main goal of the EHR Incentive Program was to increase provider use of EHRs. Although the program made big strides toward this goal, the use of EHRs is often restricted to a closed system. Despite the benefits of EHRs, considerable time and energy still need to be invested into incentivizing current EHR vendors to achieve basic interoperability with other vendors and across the multiple providers touching each patient with cancer. If the systems cannot talk with each other from a technological standpoint, data remain in silos and the potential to improve clinical care through HIT decreases. Most challenges to interoperability stem from a lack of data standardization; disparate data sources make information exchange that much more complicated. ${ }^{8}$ HIT systems must be able to efficiently communicate, exchange data, and permit healthcare professionals and patients to make effective use of the full scope of available electronic health information.

The first step toward interoperability is the transfer of medical documents from one system to another, essentially electronic movement image files
(PDFs) of medical case notes so that a provider in one institution has the medical record when the patient presents for care in the next medical institution. Transfer of data between institutions to support care can significantly improve clinical care by providing access through technology to a greater knowledge base, among other reasons. When data and reports are shared among different providers, they often must be faxed from one institution to another and then scanned into the EHR. As many as half of the documents in a patient's chart are typically scanned documents. ${ }^{3}$ In oncology, wherein most patients receive care at multiple locations from different providers, this type of report sharing can slow down providers' ability to see patients, can lead to errors or repeat testing if the scanned report cannot be found, and ultimately impacts on the quality of care received. Lack of a standard patient identifier across systems also makes data sharing and interoperability a challenge in understanding a patient's care across the continuum. ${ }^{9}$

Another component of interoperability that is proving to be a significant impediment to improving cancer care is the need for digitized data that are standardized and transferrable. If PDFs of medical case notes are transferred, then this is not digital information that can contribute to a learning health system. The information is not analyzable or able to be used for quality monitoring or research. In this instance, digitized data are needed. Efforts to digitize this unstructured data are ongoing, although proving to be manually intensive and expensive. Creating standardized data points in EHRs can allow for more seamless health information exchange that can further be used for research and comparisons across systems, organizations, and populations.

New initiatives working toward interoperability are increasing at the national and institutional level. For example, in early 2016, HHS Secretary Sylvia M. Burwell announced that companies that provide 90\% of EHRs used by US hospitals, the nation's 5 largest private healthcare systems, and more than a dozen leading professional associations and stakeholder groups pledged to implement 3 core commitments that will improve the flow of health information to consumers and healthcare providers. ${ }^{10}$ In a more localized effort, the Vanderbilt Health Affiliated Network currently has 50 hospital systems that are engaging in a health information exchange initiative. 
Although there is much work to be done in the way of interoperability, health care is far better off now compared with 20 years ago when everything was handwritten; still, there is room for improvement. ${ }^{11}$ Providers, payers, patients, and the public acknowledge the positive impact HIT can have when information exchange occurs successfully; the call for collaboration is being answered more than ever before.

\section{Using HIT to Support Alternative Payment Models}

Recent payment models have moved away from fee-for-service (quantity) payments to value-based (quality and cost) reimbursement. Rather than paying for the volume of services received, value-based payment models, referred to as APMs, often tie reimbursement to a provider's performance on specified quality measures and cost benchmarks. In order to receive payment, providers or practices must report on measures through various data sources, with the goal that quality improvement should ultimately also lead to lower costs for patients, providers, and the healthcare system. These types of payment models rely heavily on HIT, especially for reporting.

Two common complaints surrounding APMs are the measures and the reporting burden on providers to document and report on these measures. Many argue that the quality measures that exist today do not measure care that impacts outcomes; many measures are too process-oriented. Others contend that there are too many measures developed by various organizations without standardization. Providers participating in various APMs must report different measures from various data sources, creating a huge administrative reporting burden that can take time and resources away from patient care without necessarily resulting in any true quality improvement. Current methods of quality measurement typically rely on various sources of data, such as claims-based and administrative data, which often cannot provide a full picture of the care delivered. Many of the APMs require organizations to manually chart abstract in order to satisfy certain components, such as provider notes and test results, that are not documented in a structured way as part of regular standard of care. ${ }^{3}$

Reporting through the EHR greatly reduces the burden on providers and allows for standardized data points. Panelists suggest standardized, agreed-upon quality measures that can be automatically reported through the EHR. Additionally, providers should only need to enter necessary information, because if they are adding more information that is not critical to their work, the data quality declines very quickly. ${ }^{12}$ Panelists agree that it is important to be very thoughtful about exactly which data points to capture in a structured way in the clinical environment, and for stakeholders to work together to produce an agreed-upon quality list from which data can be exchanged. ${ }^{5,8}$

Another advantage to standardized measures and data capture is the ability to analyze the data on a large scale. The goal is to create data sets that can evaluate not only performance on a single measure but also how change in the measure over time aligns with patient outcomes; from there, gaps in performance can be identified and new measures that incentivize higher quality of care can be prioritized and developed. ${ }^{8}$ For example, the NCCN Outcomes Database that Flatiron Health is creating will allow for intraorganizational and interorganizational comparisons of care received. This type of research can help organizations improve care and manage their patient population.

\section{National Cancer Moonshot Initiative and the Role of HIT}

The importance of the use and interoperability of HIT is further emphasized with the announcement of the National Cancer Moonshot initiative. The National Cancer Moonshot initiative, first announced in President Obama's 2016 State of the Union address, seeks to accelerate oncology initiatives for prevention, diagnosis, treatment, and quality care. The overall goal is to achieve "a decade worth of advances" over the next 5 years by leveraging technology in the service of humanity. ${ }^{13}$ Led by Vice President Biden and the White House Cancer Moonshot Task Force, the initiative relies heavily on a public and private sector collaboration and a full governmental approach to break down HIT silos and improve patient access to quality cancer care. The National Cancer Moonshot initiative aims to provide additional resources needed to further HIT collaboration, expand clinical trials, and strengthen community oncology services. Much discussion at 
Nardi et al

the NCCN Policy Summit focused on the feasibility of achieving the Cancer Moonshot goals and the role that HIT will play in achieving these overarching priorities. According to panelists, the future success of the Cancer Moonshot will rely on breaking down data silos, leveraging data fluency, culture change, disruptive innovation, and access to data tools.

There is an opportunity like never before to make a real difference in people's lives by putting HIT in the service of patients through the EMRs, their doctors, their caregivers, and the research community; right now each of those communities has a different level of access to technology, a different level of power of their technology, and different purposes for their technology. ${ }^{9}$ A central tenant of the Cancer Moonshot is making health data interoperability a reality. Achieving widespread interoperability of health data is a well-recognized national priority that is in the best interests of patients, providers, and healthcare systems for achieving optimal costefficiencies, clinical outcomes, and patient experience, yet data remain in silos. Hence, additional challenges identified by panelists around data interoperability within the Cancer Moonshot initiative focused on digitizing, transforming, analyzing, paying for, and sharing electronic health information. Although academic medical centers are often the stewards of a large amount of health data, there are a multitude of challenges associated with storing and sharing complex health data information available in research-ready analyzable formats. Panelists identified the importance of storing health information in accessible warehouses, preferably cloud-based, with built-in analytic capabilities that allow researchers, patients, providers, and payers to access the clinical utility of the data. Data sets can link together clinical details, genomic alterations, healthcare use and costs, and additional information. Clinical decision support systems embedded in the health information can be correlated against a knowledge repository that is not isolated inside a single EHR, and can translate real-time data into actionable insights ready for use in the clinic. Data fluency, the ability to use data to make health decisions and change health practices on an individual level, poses challenges and opportunities as interoperability gets solved.

The importance of culture change to achieve widespread HIT interoperability emerged as a com- mon theme during the panel discussion. More specifically, precompetitive information, shame, game theory, and disruptive innovation were identified as critical incentives for sharing health data under the National Cancer Moonshot initiative. From a payer standpoint, the conversation shifted to the importance of adoption or acceptance of disruptive innovation when paying for quality cancer care and allowing patients access to the right treatment, at the right time, for the right price. As the standard of care accelerates from fee-for-service to value-based payment, it was argued that investments in innovation by payers will accelerate. From a commercialized data group perspective, panelists discussed the importance of standardizing a base level of information that is precompetitive, raising the overall level of health data available to the larger community.

Panelists identified advancing community oncology services as critical to achieving the Cancer Moonshot goal of driving cancer care forward. Nationally, more than $70 \%$ of cancer care occurs in a community setting. ${ }^{14}$ Although much of the advancement of cancer care occurs in academic cancer centers, there is a push to develop tools that can be used by community practitioners as seamlessly as when implemented within an academic setting. The role of HIT is to eliminate disparities at the least so that wherever a patient is, his or her doctor has the benefit of the best knowledge wherever it is. ${ }^{9}$ For example, a meaningful percentage of Foundation Medicine resources will be dedicated to the dissemination of tools for community oncologists to access clinical trials, effectively use information, and provide a platform to share health information among community cancer practices. ${ }^{15}$ Additionally, the role of decentralization in testing, access, treatment, and enrollment in clinical trials was identified as a key component to increase access within the community cancer care setting. If done effectively, the decentralization of genomic testing and data sequencing can create future opportunities for decentralized clinical input from the major academic cancer centers.

The National Cancer Moonshot initiative also aims to increase clinical trial participation to aide in new advances. An estimated $5 \%$ of adult patients with cancer participate in clinical trials ${ }^{16}$; lower participation statistics are reported for women and minorities. ${ }^{17}$ Through developing a national registry of patients with cancer interested in participating 
in clinical trials, the initiative aims to enhance the clinical trials enrollment process to allow for greater efficiency, clinical utility, and community access. Other strategies include standardizing the Institutional Review Board process, decentralizing clinical trial recruitment, and patient education efforts regarding participation in clinical trials early in a patient's evaluation and treatment process.

Overall, the Cancer Moonshot initiative seeks to increase resources and dramatically improve coordination in oncology care. Panelists concluded the session by emphasizing the importance of improving multidisciplinary cancer care coordination. Patients, advocates, communities, clinicians, researchers, legislators, payers, and government agencies must work together to accomplish the stated goals. Panelists cautioned against relying on the Cancer Moonshot as an infrastructure, but rather recommended using it as a vehicle for culture change and open data collaboration. Lastly, panelists were optimistic that the goals of the initiative can be accomplished over the next 5 years, stating that many of the strategies are currently being addressed in areas across the country, and felt that the National Cancer Moonshot will provide a common platform to advance cancer care on a wider scale to a larger audience.

\section{Precision Medicine and the Role of HIT}

Precision medicine is transforming healthcare by using genetics, genomics, and big data to move beyond one-size-fits-all models of prevention, diagnosis, and treatment. ${ }^{18}$ Moreover, personalized medicine allows the healthcare team to more effectively select treatment for patients, while improving survival outcomes and reducing adverse effects. In cancer care, genomic testing and profiling play a vital role in choosing targeted therapies based on the patient's unique tumor profile. Current estimates note that the number of genetic tests available in both oncology and non-oncology diseases is well over 2,000.19

EHRs often hold this information, although unstructured, through reports generated from the genomic testing and profiling. Given the relatively new discoveries surrounding precision medicine, the reports often hold data points that may not be actionable by the provider today. Moreover, for the average cancer practitioner, genomic testing cre- ates financial and data analysis burdens, potentially overwhelming clinical practices.

However, recent efforts aim to mitigate the overwhelming influx of genomic data for providers. Responding to Vice President Biden's Cancer Moonshot initiative, the Oncology Precision Network (OPeN) will share aggregated cancer genomics data through an advanced software platform, rapidly bringing the most promising treatment insights to patients with cancer and to providers. ${ }^{20}$ Intermountain Healthcare, Stanford Cancer Institute, Providence Health \& Services, and Syapse, Inc., a software company, announced the consortium in June 2016 with an aim to find breakthroughs in cancer care through leveraging previously untapped real-world cancer genomics data while preserving privacy, security, and data rights. This work allows OPeN to increase interoperability of data sharing, thus empowering providers with information that previously would have been unavailable.

OPeN represents one effort to advance interoperability and improve patient care through precision medicine. Such collaborations lead to greater knowledge of cancer and a higher quality of care, and can improve patient outcomes.

\section{Leveraging HIT to Improve the Quality of Clinical Care}

Intermountain Healthcare successfully champions using HIT to significantly impact clinical care despite challenges to optimization of HIT. Headquartered in Salt Lake City, Utah, Intermountain Healthcare consists of approximately 22 hospitals and 180 group practices, covering 4.5 million lives in Utah and some of the surrounding states. The organization has worked to improve the quality of clinical care through innovative methods, both from the inception of programs such as the Intermountain Precision Genomics as well as retrospectively in the Intermountain BioRepository.

Intermountain Precision Genomics comprises a personalized medicine clinic that provides patient testing and education, a molecular tumor board, drug procurement, and outcomes and cost tracking. The organization conducts its genomic testing internally so that it can track and control the data, turnaround time, and costs. Several benefits of conducting genomic testing in-house exist, including reducing the time from patient visit to test result from almost 25 
Nardi et al

days to 14 and simplifying how the results and potential treatments are presented to the oncologists and molecular tumor board. ${ }^{21}$ The molecular tumor board reviews and discusses the results and patient plans, and then the drug procurement team helps obtain the right treatment for patients.

HIT has played a critical role in Intermountain Healthcare's ability to provide a successful genomic testing program, by integrating data from multiple sources, such as laboratory, imaging, pharmacy, and analytics from different application program interfaces. Using technology from Syapse, Intermountain oncologists can view a molecular testing dashboard that shows a particular variant in a patient's cancer alongside other similar samples with that exact variant, what treatment those patients received, and what outcomes were realized.

Intermountain has used this system not only to improve patient clinical care but also to conduct research to inform care decisions. While measuring the outcomes for a cohort of patients treated within the genomic testing program and comparing their results with those of a cohort who received a standard rather than personalized medicine approach, Intermountain demonstrated statistically significant results of improved survival for patients treated with precision medicine. ${ }^{17}$ This comparison also found that the drug and total cost results of the precision medicine group were very similar to those of the standard therapy group, with the precision medicine group's mean patient costs slightly lower than those of the standard therapy group due to fewer emergency room visits and hospitalizations. ${ }^{17}$

Intermountain has also piloted a wearable device technology to improve patient outcomes. With the goal of monitoring activity and preventing unnecessary readmissions, postsurgical patients wearing the device receive messages to motivate activity; the device then monitors resulting activity and integrates the data with the remainder of the patient's medical information.

As a pioneer in the digitization space, Intermountain digitized 4 million archival samples in the Intermountain BioRepository that were saved since 1975, and is implementing high-throughput sequencing, which will allow them to combine 40 years of genomic and corresponding health outcomes data and conduct additional studies. The challenge to this type of research opportunity, however, is that the IT infrastructure necessary to ask these ques- tions, manage that data, and sift through results is immature. $^{17}$

\section{HIT and the Patient Impact}

Ultimately, HIT should be used to provide optimal care to patients with cancer. Patient characteristics and preferences make it difficult to predict the impact HIT will have on the assessment of the care received by an individual. The goal, however, is that patients will not know the breadth of HIT's impact, because the care experience will be seamless, information will be transparent and easily understandable, and patients will experience better quality of life when they feel they are receiving the best care.

HIT should be used to help optimize patientprovider time, so that providers are not spending most of their time focused on the computer screen while with their patients. The health information, therefore, needs to be easily digestible. HIT needs to be structured enough to handle disparate data but not so rigid that it does not allow for flexibility to deliver on personalized care. HIT can help foster trust in the patient-provider relationship, because it gives patients hope that the doctor is informed and not acting in isolation. ${ }^{22}$ In turn, this can help with shared decision-making, leading to patients making informed choices. Technology, it seems, should be a trust broker and accelerator. ${ }^{23}$

Disparate data sources, such as EHRs, administrative and claims data, and biologic data, used collectively, can and should be used to improve a patient's quality of life and informed decision-making. There is, however, difficulty in trying to obtain all of the data relevant to a patient's needs, particularly financial data. Further, understanding each patient's learning style and cultural background are critical, and these factors are not easily incorporated into HIT. ${ }^{24}$ There is a need to further integrate information meaningfully across sites of care, regardless of HIT system or platform; efficiently so as to optimize provider time with the patient, not detract from it; and effectively in order to offer more value-based, personalized care.

\section{Conclusions}

Speakers, panelists, and participants at the NCCN Policy Summit: Emerging Issues and Opportunities 
NCCN Policy Summit: Health Information Technology

in Health Information Technology recognized the importance of HIT in advancing cancer care for patients. HIT has had, and will continue to have, a major impact on the way patients with cancer receive care. Despite optimism and encouragement through case examples at Intermountain Healthcare and initiatives such as the National Cancer Moonshot and the Vanderbilt Health Affiliated Network, many challenges remain to optimal use of HIT, including achieving interoperability and data sharing. HIT should eliminate barriers and siloes to quality and outcomes, not create them. HIT should enable highly organized, high-quality care with credible data that are analyzable in real-time at the point of care, improve the patient-provider relationship, and empower patients to make informed decisions.

\section{References}

1. Clauser SB, Wagner EH, Aiello Bowles EJ, et al. Improving modern cancer care through information technology. Am J Prev Med 2011;40(5 Suppl 2):S198-207.

2. Mugge A. Merit-Based Incentive Payment System: Moving Beyond Meaningful Use. Presented at the NCCN Policy Summit: Emerging Issues and Opportunities in Health Information Technology; June 27, 2016; Washington, DC.

3. Electronic Health Records (EHR) Incentive Programs. Centers for Medicare $\&$ Medicaid Services. Available at: https://www.cms.gov/Regulationsand-Guidance/Legislation/EHRIncentivePrograms/index.html?redirect=/ ehrincentiveprograms. Accessed August 1, 2016.

4. EHR Incentives Programs Data Brief. November 2013. CMS. Available at: https:/www.cms.gov/regulations-and-guidance/legislation/ ehrincentiveprograms/downloads/databrief_november2013-.pdf. Accessed August 1, 2016.

5. 2014 Reporting Experience: Including Trends (2007-2015). Physician Quality Reporting System. April 15, 2016. CMS. Available at: https://www. cms.gov/Medicare/Quality-Initiatives-Patient-Assessment-Instruments/ PQRS/Downloads/2014_PQRS_Experience_Rpt.pdf. Accessed August 1, 2016.

6. Levy M. HIT: Readiness to Support Alternative Payment Models and Reporting for Precision Medicine and Quality Care. NCCN Policy Summit: Emerging Issues and Opportunities in Health Information Technology; June 27, 2016; Washington, DC.

7. Proposed Rule: Medicare Program; Merit-Based Incentive Payment System (MIPS) and Alternative Payment Model (APM) Incentive Under the Physician Fee Schedule, and Criteria for Physician-Focused Payment Models. Available at: https://www.federalregister.gov/ documents/2016/05/09/2016-10032/medicare-program-merit-basedincentive-payment-system-mips-and-alternative-payment-model-apm. Accessed August 1, 2016.

8. Shillingstad B. HIT: Readiness to Support Alternative Payment Models and Reporting for Precision Medicine and Quality Care. NCCN Policy Summit:
Emerging Issues and Opportunities in Health Information Technology; June 27, 2016; Washington, DC.

9. Roeseler A. HIT: Readiness to Support Alternative Payment Models and Reporting for Precision Medicine and Quality Care. NCCN Policy Summit: Emerging Issues and Opportunities in Health Information Technology; June 27, 2016; Washington, DC

10. HHS Announces Major Commitments From Healthcare Industry to Make Electronic Health Records Work Better for Patients and Providers. Available at: http://www.hhs.gov/about/news/2016/02/29/hhs-announces-majorcommitments-healthcare-industry-make-electronic-health-records-workbetter.html. Accessed August 1, 2016.

11. Nadauld L. HIT: Readiness to Support Alternative Payment Models and Reporting for Precision Medicine and Quality Care. NCCN Policy Summit: Emerging Issues and Opportunities in Health Information Technology; June 27, 2016; Washington, DC.

12. Abernethy A. HIT: Readiness to Support Alternative Payment Models and Reporting for Precision Medicine and Quality Care. NCCN Policy Summit: Emerging Issues and Opportunities in Health Information Technology; June 27, 2016; Washington, DC.

13. Simon G. Moonshot Initiative. NCCN Policy Summit: Emerging Issues and Opportunities in Health Information Technology; June 27, 2016; Washington, DC.

14. Vacirca J, Eagle D, Murtaugh C. Understanding Community Cancer Care: Importance of the "CARE" \& Advocating for It. Presented at the 2016 Community Oncology Conference; April 13-15, 2016; Orlando, Florida.

15. Pellini M. Moonshot: The Role of HIT. NCCN Policy Summit: Emerging Issues and Opportunities in Health Information Technology; June 27, 2016; Washington, DC.

16. Manne S, Kashy D, Albrecht T, et al. Attitudinal barriers to participation in oncology clinical trials: factor analysis and correlates of barriers. Eur J Cancer Care (Engl) 2015;24:28-38.

17. Dialogues on Diversifying Clinical Trials: Successful Strategies for Engaging Women and Minorities in Clinical Trials. Available at: http://www.fda. gov/downloads/ScienceResearch/SpecialTopics/WomensHealthResearch/ UCM334959.pdf. Accessed August 1, 2016

18. Report: Precision Medicine Already Has Measurable Impact on Patient Outcomes. HIT Consultant Web site. Available at: http://hitconsultant. net/2016/05/09/33609/. Accessed August 1, 2016.

19. American Medical Association. Genetic Testing. Available at: http:// www.ama-assn.org/ama/pub/physician-resources/medical-science/geneticsmolecular-medicine/related-policy-topics/genetic-testing.page?http:// www.ama-assn.org/ama/pub/physician-resources/medical-science/geneticsmolecular-medicine/related-policy-topic. Accessed November 23, 2015.

20. Pioneering Data-Sharing Network Created to Accelerate Cancer Precision Medicine Development. Syapse Web site. Available at: http://syapse. $\mathrm{com} / \mathrm{blog} /$ pioneering-data-sharing-network-created-to-accelerate-cancerprecision-medicine-development/. Accessed August 1, 2016.

21. Nadauld L. Leveraging HIT to Improve Quality of Clinical Care. NCCN Policy Summit: Emerging Issues and Opportunities in Health Information Technology; June 27, 2016; Washington, DC.

22. Buzaglo J. Issues, Opportunities and Patient Impact. NCCN Policy Summit: Emerging Issues and Opportunities in Health Information Technology; June 27, 2016; Washington, DC.

23. Rhee K. Issues, Opportunities and Patient Impact. NCCN Policy Summit: Emerging Issues and Opportunities in Health Information Technology; June 27, 2016; Washington, DC.

24. Edge S. Issues, Opportunities and Patient Impact. NCCN Policy Summit: Emerging Issues and Opportunities in Health Information Technology; June 27, 2016; Washington, DC. 